\title{
TROCAS: Communication Skills Development in Children with Autism Spectrum Disorders via ICT
}

\author{
Margarida Lucas da Silva ${ }^{1}$, Carla Simões ${ }^{2,3}$, Daniel Gonçalves ${ }^{1,4}$, \\ Tiago Guerreiro ${ }^{1,4}$, Hugo Silva ${ }^{1}$, and Fernanda Botelho ${ }^{3}$ \\ ${ }^{1}$ IST-UTL \\ Av. Rovisco Pais, 49, 1050-001 Lisboa, Portugal \\ margarida.lucas.silva@ist.utl.pt \\ ${ }^{2} \mathrm{FCSH}-\mathrm{UNL}$ \\ Av. de Berna, 26-C, 1069-061 Lisboa, Portugal \\ m. carla.simoes@gmail.com \\ ${ }^{3} \mathrm{ESE}$ - IPS \\ Campus do IPS, Estefanilha, 2914-504 Setúbal, Portugal \\ fernanda.botelho@ese.ips.pt \\ ${ }^{4}$ INESC-ID \\ R. Alves Redol, 9, 1000-029 Lisboa, Portugal \\ \{tjvg, daniel.goncalves\}@vimmi.inesc.pt
}

\begin{abstract}
Autism spectrum disorders (ASDs) are a triad of disturbances affecting the areas of communication, social interaction and behavior. In educational contexts, without appropriate intervention methodologies, these limitations can be deeply disabling. Our research promotes the communicative competence of children with ASDs It extends the current state-of-the-art in the field, both in terms of usability for the educators and functionality for the endusers. We performed a long-term study, and results suggest that the proposed approach is effective in promoting the development of new interaction patterns.
\end{abstract}

Keywords: Communicative competence, Autism spectrum disorder, Assistive technologies, Computer-mediated communication, Multimedia platform.

\section{Introduction}

Autism Spectrum Disorders (ASDs) are included in the global developmental disorders group, and considered to be severe and precoce neuro-development disorders that have no known cure and persist throughout life [1]. In this study, we have looked for evidence about the benefits of using a novel multimedia platform, designed specifically to develop the communicative competence in children with ASD. We have mainly focused on making it fully customizable, namely in being: (a) Appealing to the target group; (b) Easy to use both by the tutor and by the end-users; (c) Portable and easy to deploy; (d) Adaptable to the severity degree of the target users; (e) Configurable content-wise to the end-user's development stages; and (f) Evolutive according to their age and school grade.

The paper is organized as follows: Section 2 shows an overview of ASD, unveiling the need for tools to promote the communicative competences in the educational 
context, which led us to the development of a novel platform, presented in section 3 , capable of providing the best experience for children and their tutors. In sections 4 and 5 we analyze the experimental results and outline the main conclusions.

\section{Motivation}

Communication competences and social interaction are one of the areas where individuals with ASD present a higher compromise. At the education context level, an ASD frame [2] requires a set of specialized intervention measures that can attenuate the communication and integration difficulties faced by these children. Their communication abilities can be improved through specialized intervention models [3].

Currently, there is a lack of assistive technologies for the specific development of the communicative competence in children with ASDs. Existing solutions have been developed in a more enabling and functional perspective, centered in the individual himself. Examples of possible applications are: the Zac Browser, an enabler for Internet access and multimedia activities; and the Grid 2, a tool for Human-Computer Interaction. Both are aimed at different target populations.

The integration and learning difficulties faced by these children in special education schools has led to new guidelines regarding the action models to employ [4]. Currently, these children are integrated in regular education schools where they learn social inclusion. Our work is focused on a real world problem and application, and on the improvement of current practices.

\section{Proposed Approach}

Based on the practical needs of children and their tutors, we have created a fully customizable platform, with the resources that are considered beneficial for the learning environments of children with ASD, and with potential to promote the acquisition of new competences at the communication level. Under tutor supervision, the children created the overall software appearance in a way that better relates their perceived visual information with the underlying context information. From the literature revision in the field, regarding communicative competences promotion in autistic children and adequate stimuli, the platform was designed to support: (a) photos; (b) audio; (c) videos; (d) message board; (e) online digital book library access; and (f) connection to a story retell tool, MS Photostory.

All sections allows the preference classification through the "Like" and "Dislike" buttons (Fig. 1). In a very simplified way, the child can express and share his/her opinion with others, which can in turn visualize the opinions and recognize the colleagues that have expressed them. Together with the message board (Fig. 1), these are core and distinctive features of our proposed approach to the problem. The message board was developed so that users can exchange messages among themselves, recurring to writing, to symbols, or a simultaneous combination of both. The entire platform is supported by Web standards, which makes it customizable and easily adaptable to other needs that children may have. Design considerations have taken into account two different user roles: (a) end-users that use the platform; and (b) tutors that manage the platform. 


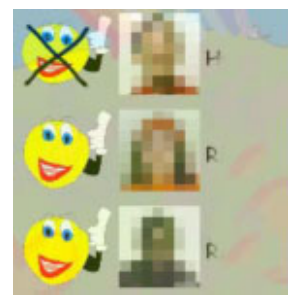

Fig. 1. Preference classification list

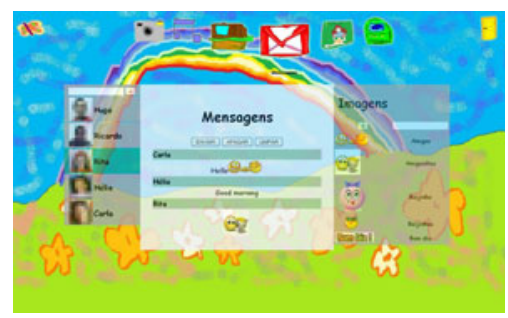

Fig. 2. Screenshot of the message board

A core aspect of the proposed platform is flexibility, that is, it can be easily adapted and customizable to the needs of each individual. Tutors can fully adjust the platform according to their goals, without the need of special knowledge regarding the use of the computer; contents are managed through the standard OS file management system, just like any other files. Accessing the TROCAS folder, the tutor can add, change, delete, and categorize the different contents through the folders. The platform automatically detects and organizes all content on the visual interfaces. Fig. 3 illustrates this process: in the folder where pictures are managed, the tutor created three categories: Animals, People, and Transportation (Fig. 3a.). The platform automatically detects these folders and organizes them on the visual interface as categories, using the name of the folder used as category descriptor (Fig. 3b.). The fact that the file names are used directly also allows the tutor to easily change the description of each content element, and facilitates the localization to other languages.

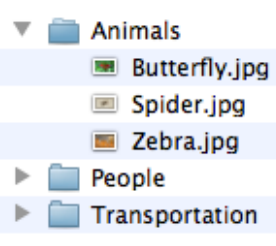

(a) Folder structure
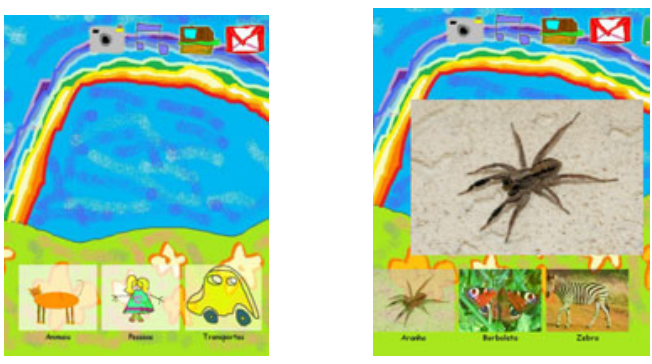

(b) Content organization in the platform

Fig. 3. Content management and categorization in the proposed platform

\section{Experimental Results}

Tests were conducted with 3 children, all with 12 years old; two of them integrated in a $5^{\text {th }}$ grade class and a third in a $6^{\text {th }}$ grade class, and all with individualized educational programs. Tutors were in charge of assessing profiles and progression according to his expert opinion. The implementation plan was: (a) a first stage of 2 weeks, which allowed the content fine tuning of the contents, platform operation, and the intervention plan; and (b) a second stage of 12 weeks, in which the established 
intervention plan was followed. Collected data revealed a consistent set of changes in the students communicative behaviours. The message board used as a way of communicating among the different agents in this process (counterparts and tutors) seems to favour the capability of understanding messages and orders. Students revealed a higher level of initiative and autonomy in exchanging messages, as well as a greater capacity of interpreting information and producing adequate responses.

It is important to enhance the fact that one of the students, in a final stage of the school year, by his/her own initiative brought a set of notes with the favorite TV characters, and asked to build a story using the resources made available by the platform. The communicative interactions between counterparts and tutors also became more frequent; the test subjects spoke frequently about the different contents of TROCAS, issuing their opinion about a given music, video, photo, etc. Of noticeable importance is the fact that one of the children, by listening to a colleague speak while viewing one of the videos expressed that: " $R$. can speak after all".

At an interpersonal relations level, the tool shows results in promotion of the relation and social interaction between counterparts. In general, students have always shown interest in using the platform, with clear increases in autonomy and proficiency not only using the platform but also using the computer, in general. Several comments and manifestations were registered, such as: (a) Requests to share TROCAS with other colleagues, tutors, and technicians; (b) Requests to use TROCAS event out of their assigned schedule; and (c) Refuse to attend classes to keep using TROCAS.

\section{Conclusions}

The use of information and communication technologies (ICT) is extremely important, as it can help reshape the teaching procedures, and make them more adequate to the reality of the subjects with special education needs. The inclusion of children with ASD in regular education schools is growing exponentially. This fact motivates the need to find new educational responses since, through adequate intervention models, the children can develop basic social competences, indispensable for an effective integration in the educational and social contexts. Experimental results collected during the period of deployment of TROCAS, show a clear relation between the intensive and guided use of the platform, and an improvement in the communicative competences among the test population. Future work will focus on implementing the tool in interfaces that may be more natural for the children.

\section{References}

1. APA: DSM.IV-TR. Quick Reference Guide for Diagnosis Criteria. Climepsi Editors (2002)

2. Jordan, R.: ASD in the Early Years: A Guide for Practitioners. QEd Pub. (2000)

3. Hewitt, S.: Specialist Support Approaches to ASD Students in mainstream settings (2005)

4. UNESCO ED-94/WS/18: The Salamanca Statement and Framework for Action on Special Needs Education. UNESCO (1994) 